\title{
Cigarette Smoking, Blood Pressure and Serum Lipids and Lipoproteins in Middle-Aged Women
}

\author{
Hiroyuki Imamura ${ }^{1)}$, Noriko Miyamoto ${ }^{1)}$, Kazuhiro Uchida ${ }^{2)}$, Kaori Teshima ${ }^{1)}$, \\ Youko Masuda $^{3)}$ and Daikichi Kobata ${ }^{4}$ \\ 1) Laboratory of Nutrition and Exercise Physiology, Department of Food and \\ Nutrition, Nakamura Gakuen University \\ 2) Department of Food and Nutrition, Nakamura Gakuen Junior College \\ 3) St. Mary's Junior College \\ 4) Department of Health Management, Minami Osaka Total Health Screening Center
}

\begin{abstract}
The relationship of cigarette smoking with blood pressure and serum lipids and lipoproteins was studied in the 3934 middle-aged women aged 40 to 59 years. After adjusting age, body mass index (BMI), alcohol intake and physical activity scores, the mean systolic and diastolic blood pressures (SBP and DBP, respectively) did not indicate dose-dependent relationships. The largest significant mean differences in SBP (4.6 mmHg), DBP (3.9 $\mathrm{mmHg}$ ), high density lipoprotein choresterol (HDL-C) (9.6 $\mathrm{mg} / \mathrm{dL}$ ), ratio of total cholesterol to HDL-C (TC/HDL-C) (0.8), triglycerides (TG) (22.9 mg/dL) and the logarithmic transformation of TG (Log TG) (0.26) were found between the non-smokers and smokers. When age, BMI, alcohol intake and physical activity scores were included in the forward stepwise multiple regression analyses, there were negative relationships found for cigarette smoking and SBP, DBP and HDL-C and positive relationships for cigarette smoking and TC/HDL-C, TG, Log TG and low density lipoprotein choresterol. Although the results are somewhat variable, the present study shows cigarette smoking is negatively associated with SBP and DBP and unfavorably associated with serum lipids and lipoproteins in middle-aged women. J Physiol Anthropol, 20 (1): 1-6, 2001 http://www.jstage.jst.go.jp/en/
\end{abstract}

Keywords: cigarette smoking, alcohol intake, blood pressure, serum lipids and lipoproteins

\section{Introduction}

The unfavorable effects of cigarette smoking on serum or plasma lipids and lipoproteins have been reported in a metaanalysis (Craig et al., 1989). The relationship between cigarette smoking and blood pressure (BP) has also been reported by Green et al. (1986) who examined the results of 15 published studies and stated that a negative association between smoking and BP was consistent over a wide range of cross-sectional epidemiological studies. However, limited number of studies have specifically examined the dose-dependent relationship between cigarette smoking and BP (Brischetto et al., 1983; Gofin et al., 1982; Green et al., 1986; Handa et al., 1990; Imamura et al., 1996; Savdie et al., 1984). Among these, a dose-dependent relationship between cigarette smoking and BP has been demonstrated in men (Handa et al., 1990; Imamura et al., 1996). However, a dose-dependent relationship was not clearly demonstrated in women (Brischetto et al., 1983; Gofin et al., 1982; Green et al., 1986), although cigarette smokers, as a group, had lower BP than non-smokers in the 2 studies (Gofin et al., 1982; Green et al., 1986).

In a recent study from our laboratory (Imamura et al., 2000), using young women, we have reported that the mean high-density lipoprotein cholesterol (HDL-C) and the ratio of total cholesterol (TC) to HDL-C (TC/HDL-C) showed dose-dependent relationships with cigarette smoking, but the mean systolic and diastolic BP (SBP and DBP, respectively) did not indicate such relationships with cigarette smoking after adjusting for age, body mass index (BMI) alcohol intake and physical activity scores. The purpose of the present study was to examine the dose-dependent relationship between cigarette smoking with BP and serum lipids and lipoproteins in middle-aged women, while taking into account several potential confounding factors such as age, BMI, alcohol intake and physical activity habits.

\section{Methods}

Subjects

The subjects of the present study were 4,422 Japanese 
women, ranging from 40 to 59 years of age. The subjects were self-referred to take physical examinations at the Minami Osaka Total Health Screening Center between August 1990 and March 1993. They were predominantly house-wives, whose socio-economic status was very similar. Of these 4,422 subjects, 488 were excluded because they were taking antihypertensive or lipidlowering drugs. Of the remaining 3,934 subjects, 3,384 (86.0\%) were non-smokers, 104 (2.7\%) were ex-smokers, and 446 (11.3\%) were smokers.

\section{Subject information and measurements}

The detail of methods in the present study have been reported elsewhere (Imamura et al., 1996). Briefly, information on smoking, drinking, and physical activity habits was obtained via a computer-administered questionnaire. The BMI was expressed as weight/height $\left(\mathrm{kg} / \mathrm{m}^{2}\right)$. Subjects were seen after an overnight fast and were asked not to smoke on the morning of the examination. The SBP and 5 th phase DBP were measured once in the morning by a trained nurse using a cuff and mercury sphygmomanometer after the subject had been sitting for more than 5 min. After BP was measured, blood was drawn from the antecubital vein. Serum lipids and lipoproteins measured were TC, HDL-C and triglycerides (TG). The blood analyzers were calibrated every morning with standard samples (Nihon Shoji, Osaka, Japan). Low-density lipoprotein cholesterol (LDL-C) was calculated using the following formula (Friedewald et al., 1972): LDL $-\mathrm{C}=\mathrm{TC}-\mathrm{HDL}-\mathrm{C}-\mathrm{TG} / 5$

\section{Statistical analysis}

Forward stepwise multiple regression analysis and analysis of covariance were performed using the general linear model of the Statistical Analysis System. Logarithmic transformation of TG (Log TG) was used to normalize a grossly skewed distribution of TG. The subjects were divided into 5 groups by their smoking habits: subjects who never-smoke, ex-smokers, and those who smoke 1-9 (light smokers), 10-19 (moderate smokers), and more than 20 (heavy smokers) cigarettes/ day. Scores of $0-4$ were given to the 5 levels of cigarette smoking. Scores of 0-2 were given to the 3 levels of physical activity (sedentary/ walk only/ play sports), and scores of 0-3 were given to the 4 levels of alcohol intake (non-drinker/ up to 1 drink/ up to 2 drinks/ more than 2 drinks per day). Forward stepwise multiple regression analysis was performed with SBP, DBP, TC, HDL-C, TC/ HDL-C, TG, Log TG, or LDL-C as the dependent variables, and cigarette smoking scores, age, BMI, physical activity scores, and alcohol intake scores as independent variables.

Differences in proportions among groups were tested using the Chi-squared test. The unadjusted mean differences among the 5 groups determined on the basis of smoking habits were determined by analysis of variance techniques. The Scheffe's method was used to identify specific significant differences when significant F-values were identified. Analysis of covariance was performed to adjust mean values for possible influences of confounding factors. A 2-sided $\mathrm{p}$ value of less than 0.05 was considered to be statistically significant.

\section{Results}

The distribution of subjects in the different smoking categories over the 4 seasons and physical activity did not differ significantly. However, smokers tended to have a higher alcohol intake than non-smokers (Table 1). Also, cigarette smoking had a significant positive correlation with alcohol intake $(\mathrm{r}=0.220 ; \mathrm{P}<0.001)$.

Table 2 presents the unadjusted mean characteristics of subjects according to smoking status. The significant mean differences among different smoking categories were observed for SBP, DBP, HDL-C, TC/HDL-C, TG, Log TG and LDL-C.

Table 3 presents the adjusted mean BP and serum lipids and lipoproteins according to smoking status. The unadjusted mean characteristics shown in the Table 2 increased after these variables were adjusted for age, BMI, alcohol intake and physical activity scores. Exsmokers and subjects in all smoking categories had significantly lower mean SBP and DBP than non-smokers. The largest mean differences in SBP (4.6 mmHg; 3.9\%) and DBP (3.9 mmHg; $5.4 \%$ ) were found between the nonsmokers and smokers. Dose-dependent relationships in both SBP and DBP were not observed after possible confounding factors were adjusted for.

The significant mean differences among different smoking categories were observed in serum lipids and lipoproteins, except TC. When non-smokers and current smokers were compared, the largest mean differences in HDL-C ( $9.6 \mathrm{mg} / \mathrm{dL} ; 15.5 \%)$, TC/HDL-C (0.8; 23.7\%), TG $(22.9 \mathrm{mg} / \mathrm{dL} ; 27.4 \%)$ and Log TG $(0.26 ; 6.0 \%)$ were found between the non-smokers and heavy smokers, and in LDL-C $(7.7 \mathrm{mg} / \mathrm{dL} ; 6.5 \%)$ was found between the nonsmokers and moderate smokers. The relationships between cigarette smoking and HDL-C, TC/HDL-C, TG, and Log TG appeared to be dose-dependent.

Table 4 presents results of the forward stepwise multiple regression analyses. There were negative relationships between cigarette smoking and SBP, DBP and HDL-C and positive relationships between cigarette smoking and TC/HDL-C, TG, Log TG and LDL-C.

\section{Discussion}

\section{Confounding factors and sampling bias}

The results of our study may not be able to be extrapolated to the entire population, because the 
Table 1 Season, Physical activity and alcohol intake

\begin{tabular}{|c|c|c|c|c|c|}
\hline & \multicolumn{5}{|c|}{ No. of cigarettes/day } \\
\hline & 0 & Ex-smoker & $1-9$ & $10-19$ & $\geq 20$ \\
\hline \multicolumn{6}{|l|}{ Season* } \\
\hline spring & $718(21.2)$ & $21(20.2)$ & $48(23.2)$ & $33(19.8)$ & $14(19.4)$ \\
\hline summer & $980(29.0)$ & $27(26.0)$ & $62(30.0)$ & $57(34.1)$ & $28(38.8)$ \\
\hline fall & $965(28.5)$ & $31(29.8)$ & $61(29.5)$ & $43(25.7)$ & $16(22.2)$ \\
\hline winter & $721(21.3)$ & $25(24.0)$ & $36(17.4)$ & $34(20.4)$ & $14(19.4)$ \\
\hline \multicolumn{6}{|l|}{ Physical activity** } \\
\hline Sedentary & 1785 (52.7) & $43(41.3)$ & $112(54.1)$ & $103(61.7)$ & $47(65.3)$ \\
\hline Walk only & 971 (28.7) & $34(32.7)$ & $56(27.1)$ & $43(25.7)$ & $14(19.4)$ \\
\hline Play sports & $628(18.6)$ & $27(26.0)$ & $39(18.8)$ & $21(12.6)$ & $11(15.3)$ \\
\hline \multicolumn{6}{|l|}{ Alcohol intake*** } \\
\hline Non-drinker & $2722(80.4)$ & $51(49.0)$ & $89(43.0)$ & $94(56.3)$ & $42(58.3)$ \\
\hline$<1$ cup / day & $583(17.2)$ & $43(41.3)$ & $91(44.0)$ & $37(22.2)$ & $10(13.9)$ \\
\hline$<2$ cup /day & $64(1.9)$ & $5(4.8)$ & $22(10.6)$ & $22(13.2)$ & $8(11.1)$ \\
\hline$\geq 2$ cup / day & $15(0.4)$ & $5(4.8)$ & $5(2.4)$ & $14(8.4)$ & $12(16.7)$ \\
\hline
\end{tabular}

Data show the number of subjects, with percentages given in parentheses. $\chi^{2}=9.12, p=\mathrm{NS} ;{ }^{* \star} \chi^{2}=21.66$, $\mathrm{p}=\mathrm{NS} ;{ }^{* * *} \chi^{2}=563.03, \mathrm{p}<0.01$.

Table 2 Subject characteristics

\begin{tabular}{|c|c|c|c|c|c|c|}
\hline \multicolumn{7}{|c|}{ No. of cigarettes/day } \\
\hline & $\begin{array}{c}0 \\
(\mathrm{n}=3384)\end{array}$ & $\begin{array}{c}\text { Ex-smoker } \\
(\mathrm{n}=104)\end{array}$ & $\begin{array}{c}1-9 \\
(\mathrm{n}=207)\end{array}$ & $\begin{array}{c}10-19 \\
(n=167)\end{array}$ & $\begin{array}{c}\geq 20 \\
(\mathrm{n}=72)\end{array}$ & $\mathrm{F}$ value \\
\hline Age (years) & $47.8 \pm 5.1$ & $46.6 \pm 5.5$ & $47.5 \pm 5.0$ & $48.2 \pm 5.6$ & $47.1 \pm 5.4$ & 2.3 \\
\hline Height (cm) & $154.7 \pm 5.3$ & $155.9 \pm 4.7$ & $154.9 \pm 4.9$ & $155.6 \pm 4.8$ & $156.0 \pm 4.8$ & 3.1 \\
\hline Weight (kg) & $54.0 \pm 7.1$ & $55.6 \pm 7.6$ & $53.0 \pm 6.5$ & $53.8 \pm 7.3$ & $54.7 \pm 8.7$ & 2.6 \\
\hline BMI $\left(\mathrm{kg} / \mathrm{m}^{2}\right)$ & $22.5 \pm 2.8$ & $22.9 \pm 2.8$ & $22.1 \pm 2.6$ & $22.2 \pm 2.8$ & $22.5 \pm 3.6$ & 2.1 \\
\hline $\mathrm{SBP}(\mathrm{mmHg})$ & $116.5 \pm 14.4$ & $113.1 \pm 14.0$ & $113.0 \pm 15.3^{*}$ & $112.6 \pm 14.3^{*}$ & $113.4 \pm 14.6$ & 7.4 \\
\hline DBP (mmHg) & $72.6 \pm 10.5$ & $70.5 \pm 9.7$ & $69.6 \pm 11.0^{*}$ & $69.2 \pm 10.5^{*}$ & $69.9 \pm 9.7$ & 9.1 \\
\hline $\mathrm{TC}(\mathrm{mg} / \mathrm{dL})$ & $198.0 \pm 33.1$ & $199.5 \pm 33.9$ & $192.7 \pm 33.6$ & $198.5 \pm 41.3$ & $194.6 \pm 39.7$ & 1.4 \\
\hline HDL-C (mg/dL) & $61.8 \pm 16.1$ & $59.6 \pm 16.6$ & $62.2 \pm 16.5$ & $55.5 \pm 16.9^{\star \ddagger}$ & $55.2 \pm 18.5^{\star \ddagger}$ & 9.0 \\
\hline TC/HDL-C & $3.41 \pm 1.05$ & $3.57 \pm 1.12$ & $3.30 \pm 1.02$ & $3.90 \pm 1.44^{\star \ddagger}$ & $3.95 \pm 1.66^{\star \ddagger}$ & 13.56 \\
\hline $\mathrm{TG}(\mathrm{mg} / \mathrm{dL})$ & $83.9 \pm 47.0$ & $91.1 \pm 41.1$ & $90.7 \pm 64.0$ & $100.1 \pm 67.2^{*}$ & $105.7 \pm 50.8^{\star}$ & 8.6 \\
\hline LogTG & $4.32 \pm 0.45$ & $4.42 \pm 0.42$ & $4.39 \pm 0.45$ & $4.48 \pm 0.47^{\star}$ & $4.56 \pm 0.44^{\star}$ & 11.17 \\
\hline LDL-C (mg/dL) & $119.4 \pm 31.4$ & $121.7 \pm 32.0$ & $112.4 \pm 31.8$ & $123.0 \pm 39.3^{\ddagger}$ & $118.2 \pm 40.5$ & 3.2 \\
\hline
\end{tabular}

Values are the mean \pm SD. The unadjusted mean differences among the 5 groups divided on the basis of smoking habits were determined by analysis of variance techniques. ${ }^{*} \mathrm{p}<0.05$ compared with non-smokers; ${ }^{\dagger} \mathrm{p}<0.05$ compared with exsmokers; ${ }^{\ddagger} \mathrm{p}<0.05$ compared with smokers smoking between one and 9 cigarettes/day. BMI, body mass index; SBP, systolic blood pressure; DBP, diastolic blood pressure; TC, total cholesterol; HDL-C, high-density lipoprotein-cholesterol; TG, triglycerides; logTG, logarithmic transformation of triglycerides; LDL-C, low-density lipoprotein-cholesterol.

subjects were self-referred, not a random sample of the general population. They were predominantly housewives of which only 446 (11.3\%) subjects were smokers. However, any bias with respect to age, BMI, physical activity, and alcohol intake have been controlled by the forward stepwise multiple regression analysis. Furthermore, the mean values of BP and serum lipids and lipoproteins were adjusted for these confounding factors by analysis of covariance. Some other confounding factors such as coffee consumption, family history of hypertension, diet, oral contraceptive use, menopause and duration of cigarette smoking may possibly influence the relationships of cigarette smoking to BP and serum lipids and lipoproteins (Green et al., 1986; Willett et al., 1983). However, no information on these factors was available in the present study.

\section{Cigarette smoking and blood pressure}

Brischetto et al. (1983), using 236 women and 214 men aged 16 to 69 years, reported that the results were similar in both sexes and did not find any relationship between cigarette smoking and BP. Gofin et al. (1982) reported that both SBP and DBP were significantly lower in smokers than in non-smokers, with greater differences in 
Table 3 Adjusted mean ( \pm SEM) of subjust characteristics



Data are the mean \pm SEM. Analysis of covariance was performed to adjust mean values for possible influences of age, body mass index, alcohol intake and physical activity. ${ }^{*} \mathrm{p}<0.05,{ }^{*} \mathrm{p}<0.01,{ }^{*} \mathrm{p}<0.001$ compared with non-smokers; ${ }^{\dagger} \mathrm{p}<0.05,{ }^{\dagger \dagger} \mathrm{p}<0.01,{ }^{\dagger \dagger} \mathrm{p}<0.001$ compared with ex-smokers; ${ }^{\ddagger} \mathrm{p}<0.05,{ }^{\ddagger \ddagger} \mathrm{p}<0.01,{ }^{\ddagger \ddagger} \mathrm{p}<0.001$ compared with smokers smoking between one and 9 cigarettes/day. SBP, systolic blood pressure; DBP, diastolic blood pressure; TC, total cholesterol; HDL-C, high-density lipoprotein-cholesterol; TG, triglycerides; logTG, logarithmic transformation of triglycerides; LDLC, low-density lipoprotein-cholesterol.

Table 4 Standardized partial regression coefficients

\begin{tabular}{|c|c|c|c|c|c|c|c|c|}
\hline & SBP & DBP & $\mathrm{TC}$ & HDL-C & TC/HDL-C & TG & LogTG & LDL-C \\
\hline Cigarette & $-0.091(0.250)^{* * *}$ & $-0.106(0.182)^{\star \star *}$ & & $-0.129(0.275)^{* \star *}$ & $0.147(0.018)^{\star \star \star}$ & $0.096(0.818)^{\star * *}$ & $0.108(0.007)^{\star * *}$ & $0.045(0.532)^{\star *}$ \\
\hline Age & $0.116(0.043)^{\star \star *}$ & $0.116(0.031)^{\star \star \star}$ & $0.301(0.098)^{* \star *}$ & & $0.163(0.003)^{* * *}$ & $0.121(0.147)^{\star * *}$ & $0.167(0.001)^{\star \star *}$ & $0.275(0.092)^{\star \star *}$ \\
\hline BMI & $0.242(0.079)^{\star \star \star}$ & $0.247(0.057)^{\star \star \star}$ & $0.126(0.180)^{* \star *}$ & $-0.305(0.086)^{* \star *}$ & $0.352(0.006)^{\star * *}$ & $0.226(0.269)^{\star * *}$ & $0.269(0.002)^{\star \star *}$ & $0.217(0.168)^{\star \star \star}$ \\
\hline Alcohol & $0.072(0.369)^{* \star *}$ & $0.083(0.268)^{\star * *}$ & $-0.076(0.809)^{\star * *}$ & $0.152(0.407)^{\star \star *}$ & $-0.179(0.026)^{\star * \star}$ & & & $-0.158(0.785)^{\star * *}$ \\
\hline $\mathrm{PA}$ & & & $0.032(0.652)^{\star}$ & $0.053(0.314)^{\star \star}$ & $-0.031(0.02)^{*}$ & $-0.036(0.975)^{\star}$ & $-0.036(0.009)^{\star}$ & \\
\hline $\mathrm{R}^{2}$ & 0.086 & 0.092 & 0.120 & 0.125 & 0.198 & 0.078 & 0.117 & 0.155 \\
\hline
\end{tabular}

Data show coefficients with the standard error given in parenthess. ${ }^{*} \mathrm{p}<0.05,{ }^{* \star} \mathrm{p}<0.01,{ }^{* \star}{ }^{\star} \mathrm{p}<0.001$. BMI, body mass index; PA,physical activity; SBP, systolic blood pressure; DBP, diastolic blood pressure; TC, total cholesterol; HDL-C, high-density lipoprotein-cholesterol; TG, triglycerides; logTG, logarithmic transformation of triglycerides; LDL-C, low-density lipoprotein-cholesterol.

middle-aged women than in men after adjusting for the effects of age, body mass, ethnic origin and season. Savdie et al. (1984) demonstrated negative dosedependent relationships between cigarette smoking and both SBP and DBP independent of drinking status and gender. However, men and women were not reported separately in this study. Ribeiro (1983) examined the joint effects of biological and social dimensions on BP by means of identifying internally homogenous subgroup of female workers and reported that lean and young smokers had significantly lower DBP than the lean and young non-smokers. However, this study did not specifically examined the dose-dependent relationships. Green et al. (1986) reported that both SBP and DBP were significantly lower in smokers than in non-smokers in the older women, but not in younger women. Among male subjects, the mean DBP was significantly lower in smokers than in non-smokers in younger men, but not in older men. In contrast, in the present study using middle aged-women, the ex-smokers and smokers had significantly lower mean SBP and DBP than in nonsmokers after adjusting age, BMI, physical activity and alcohol intake scores. The present study as well as the previous study reported from our laboratory using young women (Imamura et al., 2000) and the study by Green et al. (1986) examined dose-dependent relationships, but such relationships were not observed.

In the present study, smokers as well as ex-smokers had significantly lower mean SBP (3.8 to $4.6 \mathrm{mmHg}$ ) and DBP ( 2.9 to $3.9 \mathrm{mmHg}$ ) than non-smokers. Although it has been suggested that a $2 \mathrm{mmHg}$ reduction in DBP would result in a $17 \%$ decrease in the prevalence of hypertension as well as a $6 \%$ decrease in the risk of coronary heart disease (Cook et al., 1995), these results need to be treated with caution because, as noted by Gofin et al. (1982), it does not offset the substantial increase in coronary risk associated with smoking. However, as Green et al. (1986) stated, the role of smoking as a risk factor for hypertension is not supported by the results of this study.

The mechanisms by which chronic cigarette smoking decreases BP are unclear and need to be further elucidated. Three explanations have been proposed: (1) the alleviation of stress by cigarette smoking may 
contribute to lowering BP (Handa et al., 1990); (2) regular smokers who do not smoke before and during their examination may experience a downward rebound of BP due to the short-term absence of a nicotine stimulus (Havlik et al., 1980); (3) there may be substances in cigarette smoke other than nicotine which have a hypotensive action (Savdie et al., 1984); (4) a reduced stroke volume may contribute to lowering BP because smokers tend to have higher heart rate than non-smokers (Green et al., 1986).

\section{Cigarette smoking and serum lipids}

Brischetto et al. (1983) demonstrated positive dosedependent relationships between cigarette smoking and TC, TC/HDL-C and TG and a negative dose-dependent relationship between cigarette smoking and HDL-C after adjusting for age and weight. The results were similar in both sexes in this study. Willett et al. (1983), used women aged 20 to 40 years, reported that smokers had significantly higher TC/HDL-C and TG than non-smokers. These results did not change, even after age, weight, height, blood glucose, resting pulse, and oral contraceptive use were adjusted for. However, dosedependent relationships were not observed in this study. In a recent study from our laboratory using women aged 20 to 39 years (Imamura et al., 2000), we have reported that the mean HDL-C and TC/HDL-C showed dosedependent relationships with cigarette smoking. In contrast, in the present study, we have observed dosedependent relationships not only between cigarette smoking and HDL-C and TC/HDL-C but also between cigarette smoking and TG and Log TG after adjusting possible confounding factors. Because the experimental methods and adjusted confounding factors in the present study were the same as in the previous study (Imamura et al., 2000), the disparities in findings between these two studies may reflect differences in the subjects characteristics (i.e., middle-aged v.s. young women) and/ or in the duration of cigarette smoking.

Although the mechanisms by which cigarette smoking changes serum lipid and lipoprotein levels are not fully understood, possible explanations have been proposed by Brischetto et al. (1983). In brief, the nicotine stimulates the secretion of catecholamines as well as other hormones such as cortisol and growth hormone, leading to an increased serum concentration of free fatty acids which stimulates hepatic secretion of very low density lipoprotein and TG .

Some studies found that the mean SBP, DBP, TC, HDLC, and/or TG (Brischetto et al., 1983; Criqui et al., 1980; Gofin et al., 1982; Green et al., 1986; Muscat et al., 1991; Savdie et al., 1984; Willett et al., 1983) in ex-smokers being similar to non-smokers. In the present study, there were no significant mean differences between the two groups in TC and TG. However, the ex-smokers showed significantly lower mean SBP, DBP and HDL-C and higher Log TG, TC/HDL-C and LDL-C than non-smokers. The disparities in findings may reflect differences in the selection of subject populations and adjustments regarding confounding factors. However, with respect to BP, body weight changes may possibly affect the results. Seltzer (1974) reported that ex-smokers exhibited higher DBP over the 5-year interval when associated with weight gain, but there was no change in DBP for the ex-smokers who lost substantial amounts of body weight. Unfortunately, no information on body weight changes in different smoking categories was available in the present study.

In conclusion, the present study revealed that cigarette smoking was negatively associated with SBP and DBP, but dose-dependent relationships were not observed. The relationship of cigarette smoking to HDL-C, TC/HDL-C, TG, and Log TG appeared to be dose-dependent in the middle-aged women.

\section{References}

Brischetto CS, Connor WE, Connor SL, Matarazzo JD (1983) Plasma lipid and lipoprotein profiles of cigarette smokers from randomly selected families: Enhancement of hyperlipidemia and depression of high-density lipoprotein. Am J Cardiol 52: 675-680

Cook NR, Cohen J, Hebert PR, Taylor JO, Hennekens CH (1995) Implications of small reductions in diastolic blood pressure for primary prevention. Arch Intern Med 155: 701-709

Craig WY, Palomaki GE, Haddow JE (1989) Cigarette smoking and serum lipid and lipoprotein concentrations: An analysis of published data. Br Med J 298: 784-788

Criqui MH, Wallace RB, Heiss, G, Mishkel M, Schonfeld G, Jones GTL (1980) Cigarette smoking and plasma hightdensity lipoprotein cholesterol: The lipid research clinics program prevalence study. Circulation 62 (suppl IV): IV-70-IV-76

Friedewald WT, Levy RI, Fredrickson DS (1972) Estimation of the concentration of low-density lipoprotein cholesterol in plasma, without use of the preparative ultracentrifuge. Clin Chem 18: 499-502

Gofin R, Kark JD, Friedlander Y (1982) Cigarette smoking, blood pressure and pulse rate in the Jerusalem lipid research clinic prevalence study. Isr J Med Sci 18: 1217-1222

Green MS, Jucha E, Luz Y (1986) Blood pressure in smokers and nonsmokers: Epidemiologic findings. Am Heart J 111: 932-940.

Greene SB, Aavedal MJ, Tyroler HA, Davis CE (1977) Smoking habits and blood pressure change: a seven year follow-up. J Chronic Dis 30: 401-413.

Handa K, Tanaka H, Shindo M, Kono S, Sasaki J, Arakawa K (1990) Relationship of cigarette smoking to blood 
pressure and serum lipids. Atherosclerosis 84: 189-193

Havlik RJ, Garrison RJ, Feinleib M, Padgett S, Castelli PM, McNamara PM (1980) Evidence for additional blood pressure correlates in adults 20-56 years old. Circulation 61: 710-715

Imamura H, Tanaka K, Hirae C, Futagami T, Yoshimura Y, Uchida K, Tanaka A, Kobata D (1996) Relationship of cigarette smoking to blood pressure and serum lipids and lipoproteins in men. Clin Exp Pharm Physiol 23: 397-402

Imamura H, Uchida K, Kobata D (2000) Relationship of cigarette smoking to blood pressure and serum lipids and lipoproteins in young Japanese women. Clin Exp Pharm Physiol 27: 364-369

Muscat JE, Harris RE, Haley NJ, Wynder EL (1991) Cigarette smoking and plasma cholesterol. Am Heart J 121: 141-147

Ribeiro MBD (1983) Hypertension among female workers in Sao Paulo, Brazil: Predictors and joint effects. Hypertension 5 (supp V): V-144-V-148.

Savdie E, Grosslight GM, Adena MA (1984) Relation of alcohol and cigarette consumption to blood pressure and serum creatinine levels. J Chronic Dis 37: 617--623

Willett W, Hennekens CH, Castelli W, Rosner B, Evans D, Taylor J, Kass EH (1983) Effects of cigarette smoking on fasting triglyceride, total cholesterol, and HDLcholesterol in women. Am Heart J 105: 417-421

Received: June 30, 2000

Accepted: October 10, 2000

Correspondence to: Hiroyuki Imamura, Laboratory of Nutrition and Exercise Physiology,Department of Food and Nutrition, Nakamura Gakuen University, 5-7-1 Befu, Jonan-ku, Fukuoka 814-0198, Japan

e-mail imamura@cc.nakamura-u.ac.jp 